\title{
Virtual Nitrogen Tank to Monitor Frozen Cell Stocks
}

BioTechniques 29:122-126 (July 2000)

\author{
Arnaud Zaldumbide, Kim E. Boulukos and \\ Philippe Pognonec \\ Université de Nice, Nice, France
}

\section{RESULTS}

The program consists of seven main sections that mimic the usage of a nitrogen container: ( $i$ ) entry of vials (freezing); (ii) search of vials; (iii) information on frozen vials; (iv) removal of existing vials (thawing); (v) relocation of vials; (vi) modification of entry errors; and (vii) importing vials from another file.

These sections, described in detail below, are accessible by self-explanatory buttons. Once entered, modifications to the container content (samples and associated information) are automatically registered and limit the loss of data in case of a computer crash.

\section{Entry of Vials}

When entering the application, users are asked to identify themselves using a pop-up list that can be customized to include laboratory members. Then the user sees a list of vials that are already present in the container (Figure 1). On the screen, a prominent green button labeled Enter new vials allows users to access a window where all information concerning the vial(s) to be frozen will be entered, mainly by using customizable pop-up menus (Figure 2). Here, the user must enter at least the name of the cell line, but other parameters can be entered, such as the name of the operator (by default, the name entered when the program is opened), the number of cells present in the vials, the plate (or flask) equivalent (i.e., one-third of a 100-mm dish, etc.), the date (by default, the date when the program was opened), the number of vials frozen, the passage number of those cells and the culture medium used.

The user can also enter any relevant comments, for example, the maximum dilution of cells when split, antibiotic resistances, genetic particularities, the origin of the cells and so forth. If available, a digitized photograph of the cells (width/ height ratio $=1.5$ ) can also be pasted in a specific field. A $7.5 \times$ $5 \mathrm{~cm}$ format at a 75-dpi resolution gives high enough quality and occupies a reasonable size on the disk $(30 \mathrm{~Kb})$. After setting these parameters, the virtual vials are ready to be placed into the container. The program proposes four different choices to freeze virtual vials: (i) automatic sorting, (ii) rack restriction, (iii) box restriction and (iv) slot restriction.

The automatic sorting option distributes the vials in such a way that the first slot available (starting from rack no. 1, box no. 1) will be attributed to the first vial, the next slot available will be assigned to the second vial and so on. This choice con- 
centrates the vials as much as possible so as not to waste space and leaves any empty spaces at the end of the container.

The rack restriction option lets the user restrict the location of the vials to a specific rack. Users make their choice by viewing the graphic interface and selecting the choice that represents the racks, with those already filled indicated as such. The user simply clicks on the chosen rack and the vials

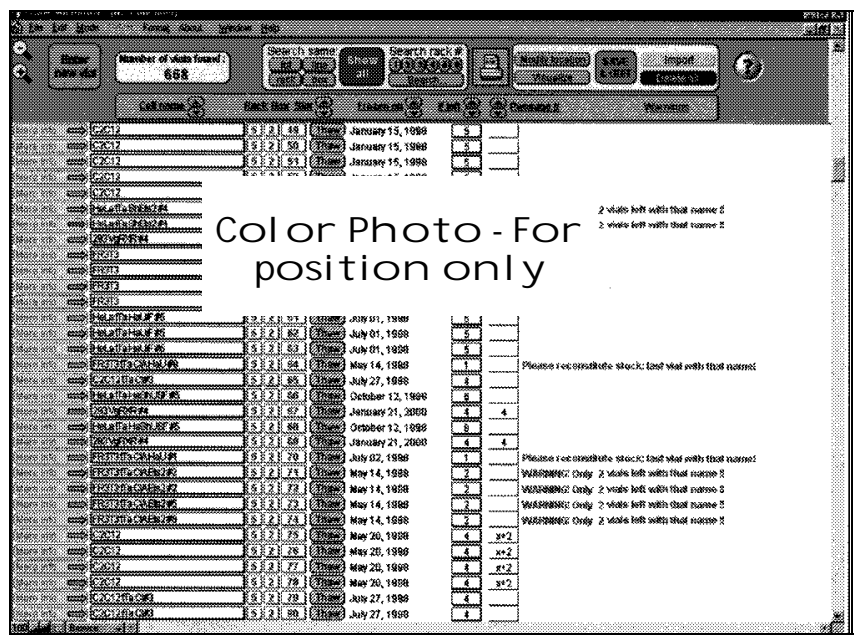

Figure 1. Main list window. This window indicates the basic information needed to identify a vial: the name of the sample, its rack, box and slot location, the date the sample was frozen, the number of sister vials present in the container and the passage number. Each lane corresponds to one vial. The righthand side displays warning messages when stocks are low. The More info button allows viewing of more detailed information (Figure 4) on the selected sample. The Thaw button allows removal of the selected vial from the container. The Enter new vial is used to add a sample to the container. Import is used to add vials coming from another FCSM file. Visualize shows a graphic representing container occupancy.

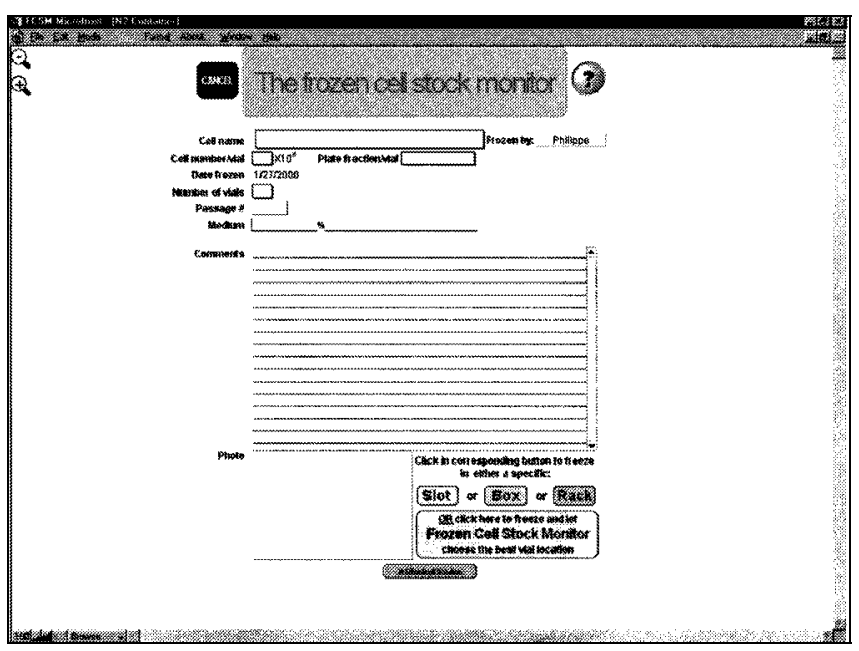

Figure 2. Sample entry window. This screen displays the different parameters that can be entered when a new sample is frozen. By default, the date is set to the date that the program is opened, and the operator is identified as the user of the program (the name is selected from a list of laboratory members when opening the application). Most of these parameters are simply chosen from customizable pop-up menus. Number of vials lets the user enter the sister (identical) vials to be frozen, in one step. Users can paste in an image of the cells using Photo. are automatically put there, with the program looking for the first available slot. This option is of particular interest to laboratories where containers are split among different groups.

The box restriction option lets the user restrict the location of the vials to a specific box. This choice is made using another graphic interface on which boxes already filled are represented as such. The user simply clicks on the chosen box, and the virtual vials will be placed automatically in that box with the program looking for the first available slot. This option will interest people who are trying to concentrate certain vials in a specific box.

The slot restriction presents the user with a graphical representation of the container. The user then clicks on the box in which the first vial is to be stored. This box is then shown open with red tubes symbolizing vials already occupying slots. The virtual vial is then stored there, after the user has clicked on the chosen free slot. If more than one vial is to be frozen, the user clicks on a second slot in the same box or selects another box in which to enter the vials, and repeats that process until the last vial is stored. When the vials are virtually frozen, a summary list of all vials harboring the exact same name will be displayed, including those just frozen.

When vials corresponding to a clone already present in the container have to be frozen, reentering all the information for that clone is not necessary. The user can simply select a vial from the clone on the list, click on the more info button and then select the green Freeze more button (Figure 4). The same screen as presented in Figure 2 will be displayed but is already filled with that cell-line name, comments, picture and so on. If necessary, this information can be freely modified. The recording process of these new vials is then absolutely identical to the one described earlier.

\section{Search of Vials}

By clicking on the search button, users will be asked to enter the specific search parameters (Figure 3). A pop-up list with cell-line names will appear but can be ignored if the search does not involve this topic. The vials can be searched using the name of the person who froze them, the date, passage number, number of vials left, growth medium used, container location, when sister vials were thawed, other associated comments and so forth. Those search parameters can be used either in combination or independently.

Ranges can be also be searched for by separating extreme values using three dots (...). For example, a sample frozen between July 4, 1776 and July 14, 1789 could be found by entering 4/7/1776...14/1789 in the Date frozen field. Vials can also be selected using the symbols < and >. In this case, samples frozen in slots smaller than 50 will be found by entering in the Slot field <50. The green Go! button will start the search, and the result, if any, will be presented in the form of a list similar to that of Figure 1.

Another way of searching the container is directly from the main list window (Figure 1). The lines present in a specific rack can be selected in one step by clicking search rack \# on the screen. Similarly, once a vial has been selected in the list (by clicking on its name, which is seen as a small black margin at the left of the more info button), the user can find sister 
vials frozen on the same date (same lot button) or all the vials from that line present in the container (same line button). It is also possible to access all the vials present in the same box (or rack) by clicking on buttons with those names, box or rack. Finally, pairs of little arrows next to the different parameters (Figure 1) allow for rapid sorting of listed vials according to the chosen criteria, either in increasing or in decreasing order.

\section{Information on Frozen Vials}

The user can now see the available information on a specific vial by clicking on the green button, more info, from the list window. A window similar to the one used to enter information during the freezing process will appear (Figure 4) and will display the information that was originally entered. In addition, a graphic of the vial location quickly identifies that particular vial when the user goes to the actual container to retrieve it.

Information and comments entered while initially freezing a vial can be seen on the screen but cannot be modified except by the supervisor of the container (see Modification of Entry Errors). This deliberate restriction avoids any modification of the original information that could be misleading to future users. However, the observation field is available, so the user can enter additional information or a correction (for example, that this line appears to be resistant to a high level of antibiotic or that a specific clone behaves differently, and so forth). When new comments have been added to the observation field, they can be transferred to the comments field, where the information is also safe from accidental modification. The date of entry and the name of the user making the update will be associated with newly added comments. This information can be added just to the vial being modified (by clicking on update this vial), to all sister vials frozen on the same date present in the container (by clicking on update all lot) or to all vials with the exact same name, including those frozen on different dates (by clicking on update this line).

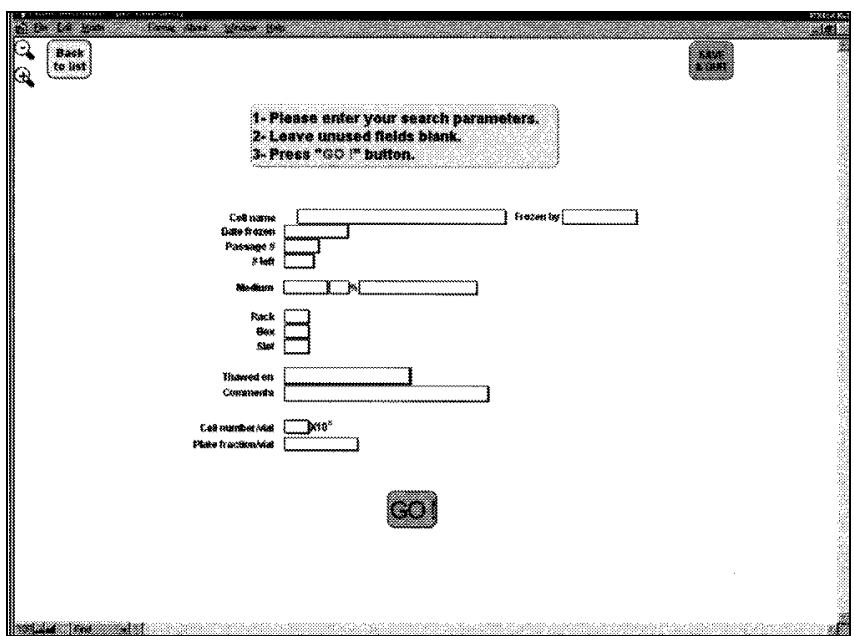

Figure 3. Search window. This screen appears when a search is performed, using either one or more search parameters simultaneously. Range searches are possible by entering the extreme values separated by three dots (...). Classical symbols such as > and < can also be entered.

\section{Removal of Existing Vials (Thawing)}

When one or more vials have been found and identified, they can be thawed, that is, removed from the list. Clicking on the red thaw button records the date, the name of the operator and the number of vials thawed. The information is put into a specific text field present in all remaining sister vials and provides a history of the vials. The operator will be warned to reconstitute the stock if the number of remaining sister vials is reduced to two or less.

If the container is being cleared of old and useless vials, thawing them individually would be tedious. Instead, the vials to be discarded, when found and listed as on Figure 1, can be removed by a click of the red Delete all button. Sister vials of the discarded ones, if any are left, are then updated to keep track of the clean-up process.

\section{Relocation of Vials}

A simple interface is available when a vial has to be moved within the container, where the actual address of the vial to be moved is displayed. Pull-down menus allow the user to modify the rack, box or slot number, thus virtually moving the vial to a new address. Of course, a new slot will have to be available for the operation to succeed. It is also possible to cancel this operation and to bring the vial to its original address.

\section{Modification of Entry Errors}

If the user enters a wrong parameter by accident while freezing a vial, it is possible to correct it in situ without using the Observations field, if the supervisor of the container

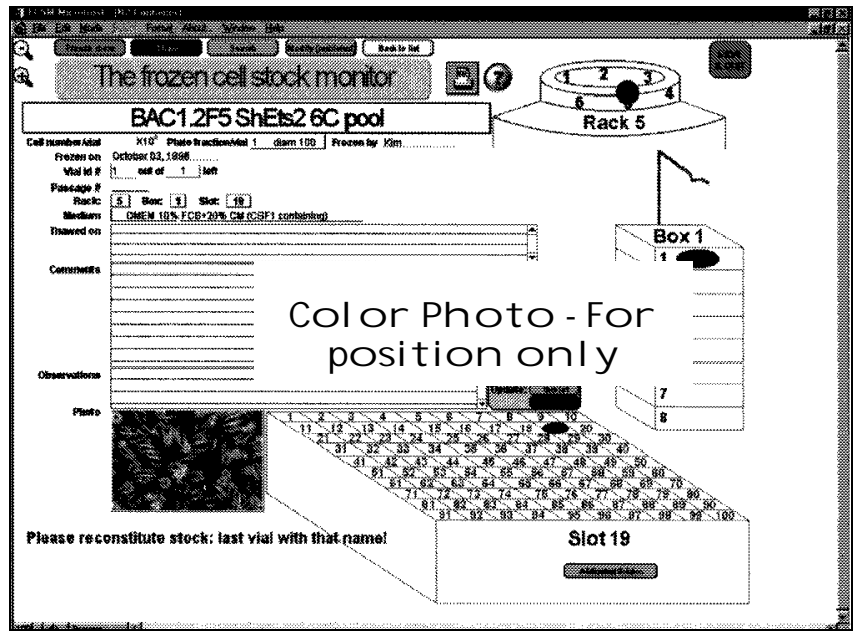

Figure 4. Vial information window. This screen appears when More information is requested for a sample. It displays all the parameters entered when freezing the sample, as well as a visual representation of the sample location in the container. The number of sister vials is shown, coupled to a warning system when stocks get low. The Thawed on field reports when sister vials have been thawed, and by whom. The Observations field is used when additional information must be entered and is safely recorded in the Comments field. The date of this update and the name of the author are automatically included. Modify (restricted) gives access to a password-protected window where these parameters can be directly modified. 
agrees with the correction. This is particularly useful when a line name has been misspelled. In such a case, the program will not recognize the same line frozen on a different date because of name differences (a single extra space in the name would be enough), and this could lead to incorrect stock information. Similarly, if an incorrect photograph is pasted, future users could be confused. Thus, users can access a passwordrestricted window by clicking the button, Modify (restricted) (Figure 4). Each parameter can then be modified directly.

To simplify the modification of many vials, arrows on each side of the cell-line name allow access to the previous or next vial. Even though this feature is useful, it can be dangerous because entering the wrong parameters may interfere with the tracking of the vials.

\section{Importation of Vials from Another File}

In a laboratory where a container is shared among several users, we recommend that each user be given a copy of the program. Users would then enter their own vials in their personal box or rack, with no risk of confusion with their colleagues' samples. When a user leaves, the supervisor can import or take the box or rack of that user's frozen samples and place them in a virtual container. This is done by clicking on the Import button, shown in the window of Figure 1.

After entering a password, the container supervisor chooses the option, Import vial, and selects the other virtual container from which samples have to be imported. By double clicking, the moving of vials will start. When the process is complete, the imported vials will be part of the receiving container. If one or more of the imported vials' slot addresses are already occupied in the receiving container, a list displays all the slots that are doubly occupied and indicates which are original and which are extraneous. Then, it is possible to either delete all original vials that as a group, share a slot with imported ones or to delete all the imported vials that as a group have been put in slots already occupied. It is also possible to delete vials one by one and thus to choose the original vial in one case and the imported vial in another.

If only part of the exporting container needs to be moved (for example, box 2, rack 3), the operator selects these samples in the exporting container using the search mode and search parameters, box 2 and rack 3 . When these and only these samples are listed in the exporting container, the user simply closes it, goes back to the virtual container and carries out the movement as described earlier. Only the selected vials are moved.

\section{DISCUSSION}

The FCSM is a 4800-vial virtual container consisting of six racks, each holding eight 100-slot boxes. Using it, laboratories can efficiently track all their frozen samples throughout time and despite the high turnover of personnel associated with laboratories. It is interactively easy to use. The program, developed on FileMaker Pro ${ }^{\mathrm{TM}}$, runs on both PCs and Macintosh computers, and its runtime binding engine will run without having to install FileMaker Pro on the receiving computer.

The unbound FileMaker Pro version is also available upon request if a laboratory wants to merge it with another existing database or modify it to fit another container type. Online help is available for newcomers, which eliminates any timeconsuming training for new workers.

To avoid accidental loss of data, we recommend that every user have their own program, installed on different computers to which only personal vials will be entered in allotted container space. When necessary, principal investigators can easily select and import vials of interest into their own virtual container. The program also offers an option on the opening window to request an automatic backup every time the application is closed. This requires double-disk occupancy but ensures that the data will not be lost, even in case of accidental file destruction. Similar to other critical data, we recommend saving the copy in a different place.

Printouts of detailed information corresponding to individual vials, as well as lists of selected vials, can be obtained by clicking on the Print icon on the appropriate screen. The occupancy status of a box can also be printed graphically, verifying in a glance that the actual occupancy of the real box corresponds to the virtual one.

Finally, we recommend running this application on a computer configuration with at least $64 \mathrm{MB}$ RAM, $40 \mathrm{MB}$ free disk space and a $400 \mathrm{MHZ}$ (or G3 on a Macintosh) processor. A 15 -inch monitor $(1024 \times 768$ pixels $)$ is recommended for easy viewing of the program.

This program will be licensed free to any academic laboratory that requests it. Send your request by e-mail to pognonec@unice.fr and indicate FCSM in the subject field.

\section{ACKNOWLEDGMENTS}

We are indebted to Dr. Jean Claude Chambard for his help and suggestions, as well as for the time he devoted beta-testing this application. A.Z. is supported by a doctoral fellowship from the Ministere de la Recherche et de la Technologie, K.E.B. and P.P. are supported by CNRS. Grant support was provided by A.R.C. (no. 9691 and no. 5231) to K.E.B.

\section{REFERENCE}

1.Nayler, O. and S. Stamm. 1999. ScienceLabDatabase: a computer program to organize a molecular biology laboratory. BioTechniques 26:11861191.

Received 12 November 1999; accepted 2 March 2000. 